\title{
Identification of key mRNAs and microRNAs in the pathogenesis and progression of osteoarthritis using microarray analysis
}

\author{
HUA LI $^{1 *}$, BING BAI $^{2 *}$, JINGRUI WANG $^{3}$, ZANMEI XU $^{3}$, SAIYING YAN $^{3}$ and GUOBIN LIU ${ }^{4}$ \\ ${ }^{1}$ Department of Orthopedics, Harrison International Peace Hospital, Hengshui, Hebei 053000; \\ ${ }^{2}$ Department of Orthopedics, Linxi People's Hospital, Xingtai, Hebei 054900; ${ }^{3}$ Tianjin Marvelbio \\ Technology Co., Ltd., Lingao Creative Industry Park, Nankai, Tianjin 300381; ${ }^{4}$ Department of Orthopedics, \\ The First Hospital of Hebei Medical University, Shijiazhuang, Hebei 050000, P.R. China
}

Received November 1, 2016; Accepted June 14, 2017

DOI: $10.3892 / \mathrm{mmr} .2017 .7251$

\begin{abstract}
Osteoarthritis (OA) is a common type of disease affecting the joints that results from the breakdown of joint cartilage and the underlying bone; currently, its pathogenesis is still unclear. The aim of the present study was to identify key mRNAs and miRNAs involved in the pathogenesis and progression of OA using microarray analysis. The gene expression profile of GSE27492 was downloaded from the Gene Expressed Omnibus database, and included 49 arthritic mouse ankle samples collected at 6 time points $(0,1,3,7,12$ and 18 days) following the induction of arthritis via serum transfer. Differentially expressed genes (DEGs) were identified in ankle samples taken on days 1,3, 7, 12 and 18 following serum transfer compared with day 0 samples, and overlapping DEGs in day 3, 7, 12 and 18 samples were identified. The Database for Annotation, Visualization and Integrated Discovery online tool was used to perform functional and pathway enrichment analyses of the overlapping DEGs. The miRWalk database was used to identify potential micro (mi) RNAs regulating the selected overlapping DEGs, and regulatory miRNA-target mRNA pairs were obtained. The Cytoscape platform was used to establish and visualize the miRNA-mRNA regulatory network. The present results revealed that 35, 103, 62 and 75 DEGs were identified in day 3, 7, 12 and 18 samples, respectively. A total of 17 overlapping DEGs were identified among the 4 sample sets, and revealed to be enriched in 14 gene ontology terms and 3 Kyoto Encyclopedia of Genes and Genomes pathways. miRWalk analysis identified 242 potential miRNA-mRNA regulatory pairs and 211 nodes were revealed
\end{abstract}

Correspondence to: Dr Guobin Liu, Department of Orthopedics, The First Hospital of Hebei Medical University, 89 Dong-Gang Road, Shijiazhuang, Hebei 050000, P.R. China

E-mail: bugeng@126.com

${ }^{*}$ Contributed equally

Key words: osteoarthritis, microarray, Gene Expressed Omnibus, Database for Annotation, Visualization and Integrated Discovery to be involved in the miRNA-mRNA regulatory network. The present study identified potential genes, including C-type lectin domain family 4 member $\mathrm{D}$, chemokine (C-X-C motif) ligand 1 and $\mathrm{C}-\mathrm{C}$ motif chemokine ligand, and pathways, including chemokine signaling pathways, cytokine-cytokine receptor interactions and nucleotide-binding oligomerization domain-like receptor signaling pathways, which may be involved in the pathogenesis and progression of OA. These findings may help elucidate the molecular mechanisms underlying OA pathophysiology, and may be useful for the development of novel therapeutic targets for the treatment of patients with OA.

\section{Introduction}

Osteoarthritis (OA), also known as wear-and-tear arthritis or osteoarthrosis, is a degenerative joint disease and the most common form of arthritis (1). The incidence of OA increases with age, and obesity is also a risk factor for the disease (2-4). In the USA, $\sim 27$ million patients with OA have been reported, and the prevalence of the disease is significantly enhanced at ages $>50$ years for males and $>40$ years for females $(5,6)$. According to the American College of Rheumatology, 70\% of people $>70$ years of age exhibit X-ray evidence of OA (7). As the population ages, it has been estimated that $20 \%$ of Americans will be over the age 65 years by 2030 (8), thus raising the risk for developing OA.

OA is caused by the breakdown of cartilage in one or more joints, and its symptoms include joint pain, stiffness, swelling, tenderness and inflexibility (9). Currently available therapeutic strategies for the treatment of OA include lifestyle modifications (e.g. exercise and weight loss), analgesics and sometimes surgical intervention (10); the primary focus of $\mathrm{OA}$ treatment is the reduction of pain and improving the function of the affected joints. OA is a chronic condition and its pathogenesis involves the interaction of multiple factors including genetic, metabolic, biochemical and biomechanical factors (11). Berenbaum et al (12) suggested that OA may be caused by mechanical stress on the joint and an ongoing low-grade inflammatory response. Histone deacetylase 4 has been demonstrated to contribute, at least in part, to the mechanisms underlying cartilage degeneration during OA 
pathogenesis (13). Several genes have been associated with the development and progression of OA $(14,15)$; however, the mechanisms underlying the pathophysiology of the disease have yet to be fully elucidated, whereas the need for more effective and highly specific therapeutic strategies for the treatment of patients with OA is urgent.

In the present study, a set of bioinformatics approaches were used to comprehensively analyze the microarray data from mice with serum-transferred arthritis, publicly available at the Gene Expression Omnibus (GEO) database. Differentially expressed genes (DEGs) were identified, and functional and pathway enrichment analyses were performed, in order to identify novel biomarkers and suggest putative mechanisms that may be involved in the pathogenesis and progression of OA.

\section{Materials and methods}

Microarray data. The microarray data of GSE27492 was downloaded from the GEO database (www.ncbi.nlm.nih. gov/geo/), which was provided by Jacobs et al (16). The dataset included 49 arthritic mouse ankle samples collected at 6 time points $(0,1,3,7,12$ and 18 days, including 7, 5, 9, 11, 9 and 8 samples, respectively) following the induction of arthritis via serum transfer. Microarray data obtained from GSE27492 were sequenced on the platform of GPL81 [MG_U74Av2] Affymetrix Murine Genome U74A 2.0 Array (Affymetrix; Thermo Fisher Scientific, Inc., Waltham, MA, USA).

Data preprocessing. The original raw data were converted into a format recognizable by the $\mathrm{R}$ statistical software program and scripting language, and the Affy v1.40.0 package (http://www.bioconductor.org/packages/2.13/bioc/html/affy. html) (17) was used for background correction and quartile normalization. This was followed by the conversion of probe symbols to gene symbols; if multiple probes corresponded to a single gene symbol, the average expression value of the probes was used as the expression value of the gene.

Screening of DEGs. The comparison of DEGs was performed using the Limma v3.18.13 package (http://www.bioconductor. org/packages/2.13/bioc/html/limma.html) (18) on $\mathrm{R} ; \mathrm{P}<0.05$ and $\log _{2}$ (fold change) $\mid>1$ were defined as the cut-off values for screening. A total of 5 sets of DEGs were detected, including DEGs compared between ankle samples obtained on days 1,3, 7,12 and 18 post-serum injection and ankle samples obtained on day 0; these were denoted as DEGs-1d, DEGs-3d, DEGs-7d, DEGs-12d and DEGs-18d, respectively. The 5 DEG sets were compared and the overlapping DEGs were identified and used for subsequent analyses.

Functional and pathway enrichment analysis. The Database for Annotation, Visualization and Integrated Discovery (DAVID) online tool (https://david.ncifcrf.gov/) (19) is used for systematically associating the functional terms with gene or protein lists. In the present study, DAVID was used to perform Gene Ontology (GO) and Kyoto Encyclopedia of Genes and Genomes (KEGG) pathway enrichment analysis of the previously identified overlapping DEGs. $\mathrm{P}<0.05$ was considered the cut-off value. micro (mi) RNA target prediction. The miRWalk database version 2.0 (http://www.umm.uni-heidelberg. de/apps/zmf/mirwalk/) (20) is a powerful and accurate database that displays miRNAs and their corresponding target genes and binding sites in mice, rats and humans. In the present study, miRNAs regulating the identified overlapping DEGs were predicted based on the information on miRWalk. Putative targets were predicted by $>5$ bioinformatics algorithms among the 10 algorithms in the miRWalk database: DIANA-microT v4.0 (http://diana.imis. athena-innovation.gr/DianaTools/index.php?r=microT_CDS/ index), miRanda-rel2010 (http://www.microrna.org/ microrna/getDownloads.do), miRDB v4.0 (http://mirdb. org/miRDB/download.html), miRWalk v2.0 (http://zmf. umm.uni-heidelberg.de/apps/zmf/mirwalk2/), RNAhybrid v2.1 (https://bibiserv.cebitec.uni-bielefeld.de/rnahybrid/ dl_pre-page.html), TargetScan v6.2 (http://www.targetscan. org/cgi-bin/targetscan/data_download.cgi?db=vert_61),RNA22 v2.0 (https://cm.jefferson.edu/rna22/), PITA (https://genie. weizmann.ac.il/pubs/mir07/mir07_exe.html), PICTAR5 and PICTAR4 (http://pictar.mdc-berlin.de/).

Construction and analysis of the miRNA-mRNA regulatory network. After the miRNA-mRNA regulatory pairs were identified, the miRNA-mRNA regulatory networks were constructed and visualized using Cytoscape v3.5.1 software (http://www.cytoscape.org/download.php) (21). In addition, nodes were analyzed according to the intimate connections with other nodes. The important nodes in the network where identified when the degree of node attributes was $\geq 1$, where 'degree' represented the connections with other nodes. In the regulatory network, a circular node represented the miRNA and a hexagonal node represented the mRNA.

\section{Results}

DEGs in OA. A total of 35 (28 up- and 7 downregulated), 103 (81 up- and 22 downregulated), 62 (53 up- and 9 downregulated) and 75 (67 up- and 8 downregulated) DEGs were identified among the DEGs-3d, DEGs-7d, DEGs-12d and DEGs-18d sets, respectively; no DEGs were identified in the DEGs-1d set. The numbers of the DEGs within the DEGs-3d, DEGs-7d, DEGs-12d and DEGs-18d sets are presented in Fig. 1. The number of DEGs appeared to reach a maximum on day 7 following the initial serum injection. In addition, a Venn diagram was constructed for the 4 DEG sets to display the overlapping genes, and is presented in Fig. 2. A total of 17 overlapping DEGs were detected among the aforementioned 4 DEG sets, and are listed in Table I. Among those, the expression of 16 genes was upregulated and the expression of 1 gene was downregulated.

GO terms annotation and KEGG signaling pathway enrichment of the overlapping DEGs. Functional and pathway enrichment analyses were performed for the 17 overlapping DEGs. As presented in Table II, the overlapping DEGs were significantly enriched in $14 \mathrm{GO}$ terms. The majority of the GO terms were associated with immune processes, including immune responses, response to injury and inflammatory responses.

KEGG enrichment analysis was performed to understand the signaling pathways of DEGs involved in OA. The 17 
Table I. Overlapping differentially expressed genes in arthritic ankle samples obtained on days 3, 7, 12 and 18 following the initial serum injection, compared with ankle samples isolated on day 0 .

\begin{tabular}{|c|c|c|c|c|c|}
\hline Gene & $\log \mathrm{FC} \_3 \mathrm{~d}$ & $\log \mathrm{FC}_{-} 7 \mathrm{~d}$ & $\log \mathrm{FC} \_12 \mathrm{~d}$ & $\log \mathrm{FC} \_18 \mathrm{~d}$ & Regulation type \\
\hline AF251705 & 1.305238 & 1.457843 & 1.423637 & 1.534935 & up \\
\hline Adam8 & 1.397521 & 1.545198 & 1.64087 & 1.397731 & up \\
\hline Arg 1 & 3.216408 & 3.470759 & 3.253301 & 3.500893 & up \\
\hline Basp1 & 1.183961 & 1.402966 & 1.330427 & 1.417313 & up \\
\hline $\mathrm{Ccl} 2$ & 3.136399 & 2.754264 & 2.608425 & 2.605791 & up \\
\hline $\mathrm{Ccl} 7$ & 2.379615 & 1.747449 & 1.676018 & 1.364921 & up \\
\hline Ccl9 & 1.592837 & 1.25133 & 1.348077 & 1.222959 & up \\
\hline Ccr2 & 1.395741 & 1.265384 & 1.07037 & 1.021065 & up \\
\hline $\mathrm{Clec} 4 \mathrm{a} 2$ & 1.344762 & 1.371277 & 1.486593 & 1.286852 & up \\
\hline Clec4d & 2.328534 & 3.102078 & 3.12611 & 3.014611 & up \\
\hline Cxcl1 & 1.152963 & 2.378911 & 2.050006 & 2.15751 & up \\
\hline Ephx1 & -1.12827 & -1.5318 & -1.36148 & -1.05361 & down \\
\hline Fabp5 & 1.300109 & 1.492187 & 1.360936 & 1.174031 & up \\
\hline Fcgr1 & 1.565833 & 1.337319 & 1.266228 & 1.255231 & up \\
\hline Gp49a & 1.08629 & 1.48513 & 1.472467 & 1.684876 & up \\
\hline Il1rn & 2.356432 & 2.933974 & 2.905693 & 3.029609 & up \\
\hline Saa3 & 2.536273 & 3.692907 & 3.451593 & 4.212274 & up \\
\hline
\end{tabular}

FC, fold change.

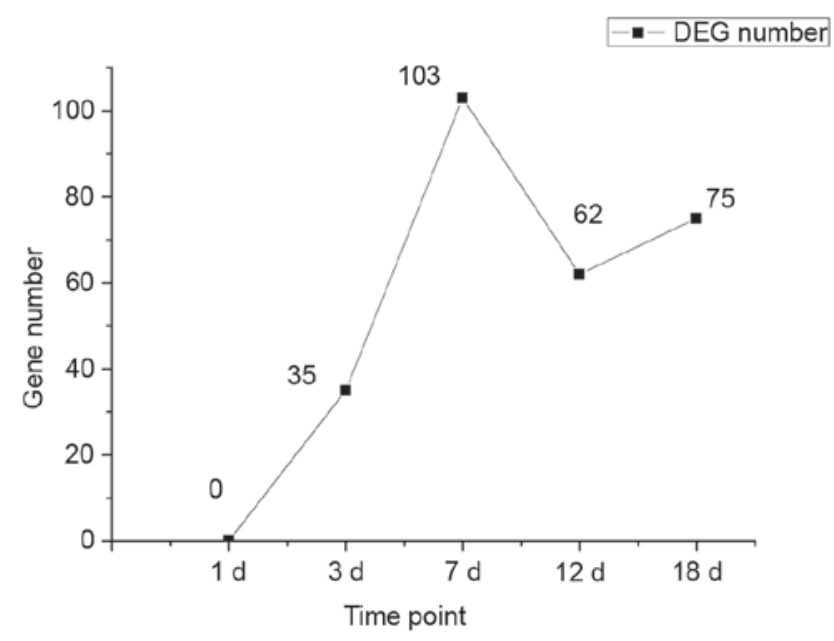

Figure 1. DEG numbers within the DEGs-3d, DEGs-7d, DEGs-12d and DEGs-18d sets. DEGs were compared between ankle samples obtained on days $1,3,7,12$ and 18 post-serum injection and ankle samples obtained on day 0, and denoted as DEGs-1d, DEGs-3d, DEGs-7d, DEGs-12d and DEGs-18d, respectively. No DEGs were identified between samples obtained on days 1 and 0 . DEG, differentially expressed gene; $d$, day.

overlapping DEGs were revealed to be enriched in 3 KEGG signaling pathways, including chemokine signaling pathway, cytokine-cytokine receptor interaction and nucleotide-binding oligomerization domain (NOD)-like receptor signaling pathway (Table III).

Construction of the miRNA-mRNA regulatory network. A total of 242 miRNA-mRNA regulatory pairs were predicted using the miRWalk database, and, according to the predicted

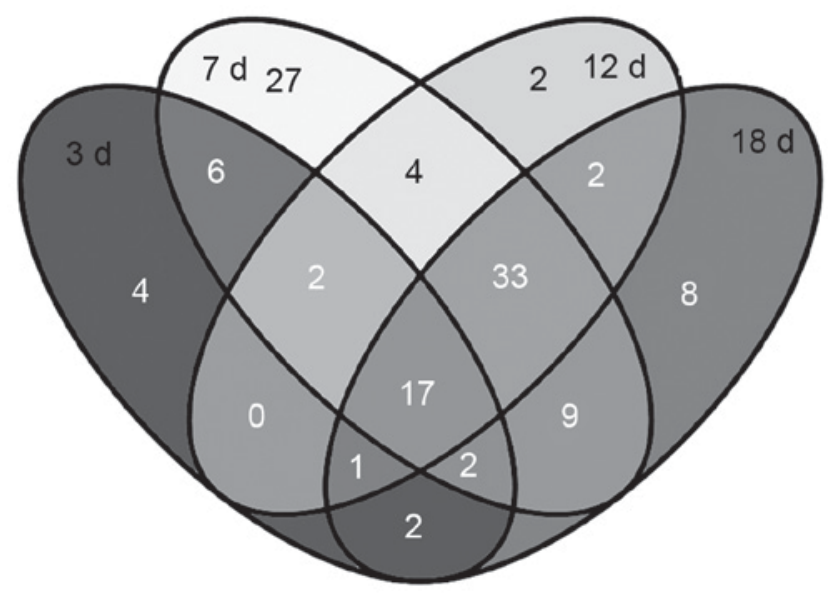

Figure 2. Venn diagram for the 4 DEG sets: DEGs-3d, DEGs-7d, DEGs-12d and DEGs-18d. A total of 17 overlapping DEGs were detected among the 4 aforementioned DEG sets. DEG, differentially expressed gene; d, day.

database number, the top 40 most significant pairs are listed in Table IV. The miRNA-mRNA regulatory network is presented in Fig. 3, as constructed using Cytoscape software, and the 40 most significant nodes, listed in Table $\mathrm{V}$, all had higher degrees, which reflects their intimate connections with other nodes. A total of 211 nodes composed the miRNA-mRNA regulatory network, including 9 mRNAs and 202 miRNAs (Fig. 3).

\section{Discussion}

$\mathrm{OA}$ is a polygenic disorder, and a genetic contribution serves a critical role in the development and progression of the 
Table II. GO enrichment pathways of the overlapping differentially expressed genes.

\begin{tabular}{llcll}
\hline Category & \multicolumn{1}{c}{ GO term } & Gene number & P-value & \\
\hline BP & Immune response & 11 & $5.86 \times 10^{-12}$ & CXCL1, CCL2, GP49A, CCR2, IL1RN, CCL9, \\
& & & & CLEC4A2, AF251705, CLEC4D, FCGR1, CCL7 \\
BP & Response to injury & 7 & $1.09 \times 10^{-6}$ & CXCL1, ARG1, CCL2, CCR2, SAA3, FCGR1, \\
& & & CCL7 \\
BP & Inflammatory response & 6 & $3.12 \times 10^{-6}$ & CXCL1, CCL2, CCR2, SAA3, FCGR1, CCL7 \\
MF & Chemokine activity & 4 & $4.66 \times 10^{-6}$ & CXCL1, CCL2, CCL9, CCL7 \\
MF & Chemokine receptor binding & 4 & $5.05 \times 10^{-6}$ & CXCL1, CCL2, CCL9, CCL7 \\
CC & Extracellular space & 7 & $1.65 \times 10^{-5}$ & CXCL1, ARG1, CCL2, IL1RN, SAA3, CCL9, CCL7 \\
$\mathrm{BP}$ & Defense response & 6 & $8.70 \times 10^{-5}$ & CXCL1, CCL2, CCR2, SAA3, FCGR1, CCL7 \\
$\mathrm{CC}$ & Extracellular region part & 7 & $1.70 \times 10^{-4}$ & CXCL1, ARG1, CCL2, IL1RN, SAA3, CCL9, CCL7 \\
$\mathrm{MF}$ & Cytokine activity & 4 & $4.92 \times 10^{-4}$ & CXCL1, CCL2, CCL9, CCL7 \\
$\mathrm{BP}$ & Chemotaxis & 3 & 0.006254 & CCL2, CCL9, CCL7 \\
$\mathrm{BP}$ & Taxis & 3 & 0.006254 & CCL2, CCL9, CCL7 \\
$\mathrm{CC}$ & Extracellular region & 7 & 0.009876 & CXCL1, ARG1, CCL2, IL1RN, SAA3, CCL9, CCL7 \\
$\mathrm{BP}$ & Locomotor behavior & 3 & 0.027823 & CCL2, CCL9, CCL7 \\
$\mathrm{MF}$ & Carbohydrate binding & 3 & 0.031976 & CLEC4A2, CLEC4D, CCL7 \\
\hline
\end{tabular}

GO, Gene Ontology; BP, biological process; MF, molecular function; CC, cellular component.

Table III. Enriched Kyoto Encyclopedia of Genes and Genomes pathways of the overlapping differentially expressed genes.

\begin{tabular}{llrr}
\hline Category & \multicolumn{1}{c}{ Pathway Name } & Count & P value \\
\hline KEGG_PATHWAY & Chemokine signaling pathway & 4 & $8.21 \times 10^{-4}$ \\
KEGG_PATHWAY & Cytokine-cytokine receptor interaction & 4 & $1.57 \times 10^{-3}$ \\
KEGG_PATHWAY & NOD-like receptor signaling pathway & 2 & $8.19 \times 10^{-2}$ \\
\hline
\end{tabular}

KEGG, Kyoto Encyclopedia of Genes and Genomes; NOD, nucleotide-binding oligomerization domain.

disease $(22,23)$. Loughlin (23) suggested that the genetic risk for OA may primarily result from alterations in gene expression modulation, an effect typically mediated though the regulation of transcription. In the present study, no DEGs were detected in ankle samples obtained on day 1 following the initial serum injection, compared with in day 0 samples. These findings suggested that serum-transferred arthritis may be developed at time points later than 1 day post-injection, or that the induced OA may not alter gene expression compared with normal ankle tissue at this early time point. Conversely, the number of DEGs reached its maximum value on day 7 following the initial serum injection, thus indicating that 7 days following serum transfer, the development of OA was ongoing. Subsequently, the number of DEGs appeared to be decreased on days 12 and 18 post-injection.

Previous research has demonstrated that various types of cells, cytokines and chemokines, the complement cascade, and other immune processes are involved in the pathogenesis of OA (24-26). Wang et al (27) reported that complement expression and activation were significantly enhanced in OA synovial tissue, particularly during the early stages of OA pathogenesis. Kandahari et al (28) have reviewed and summarized the roles of the innate and adaptive immune response in early OA pathogenesis. There were less genes in the DEG sets, DEGs-12d and DEGs-18d, when compared with DEGs-7d, which may be associated with the suppression of the autoimmune system. In addition, similar alterations in gene expression were detected among the 17 overlapping DEGs (16 up- and 1 downregulated) in the 4 DEG sets (DEGs-3d, DEGs-7d, DEGs-12d and DEGs-18d) that were examined. These findings suggested the overlapping DEGs may serve critical roles in the pathogenesis and progression of OA.

The overlapping DEGs were enriched in 14 GO terms, including immune, injury and inflammatory response pathways, chemokine activation pathways and chemokine receptor binding. The majority of the identified pathways were associated with the processes of immunity and inflammation. OA is considered an inflammatory disease (11), and the implication of immune responses in the pathogenesis of OA has previously been studied (29). Synovial inflammation, immune cell activation and proinflammatory cytokine production have been demonstrated to participate in the pathogenesis and progression of OA (23), whereas anti-cytokine therapy has been suggested as an effective therapeutic approach for the treatment of patients with OA (30). Therefore, the GO terms that 


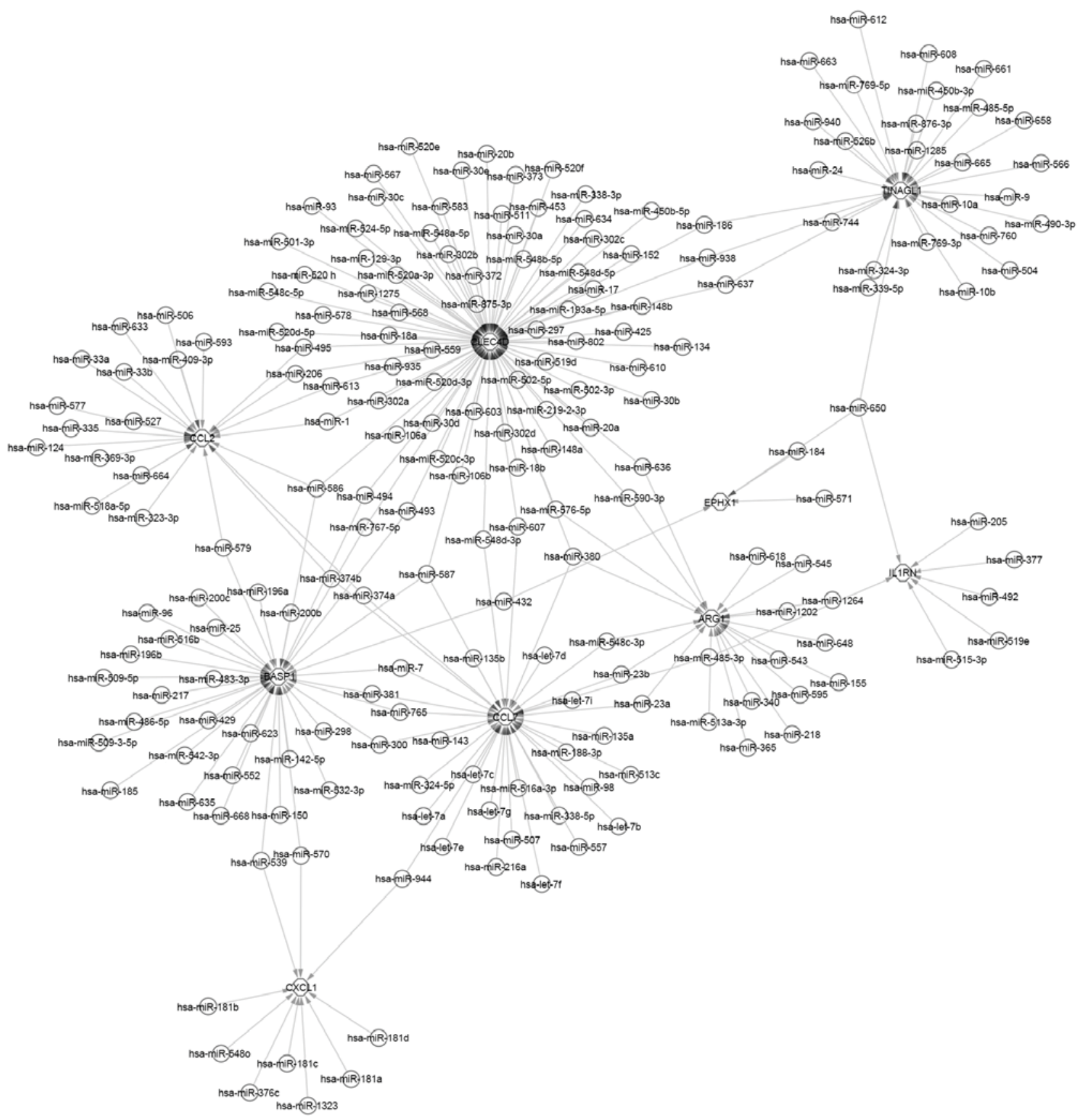

Figure 3. miRNA-mRNA regulatory network for the overlapping DEGs. In the regulatory network, a circular node represents miRNAs and a hexagonal node represents mRNAs. A total of 211 nodes composed the miRNA-mRNA regulatory network, including 9 mRNAs and 202 miRNAs. mi, micro; DEG, differentially expressed gene.

were identified in the present study may be closely associated with the pathogenesis and progression of $\mathrm{OA}$, and may provide useful indicators for the development of treatment strategies for patients with OA.

Chemokine (C-X-C motif) ligand $1(C X C L 1)$ and $\mathrm{C}-\mathrm{C}$ motif chemokine ligand 2 (CCL2) were revealed to be involved in most of the GO terms that were investigated. $C X C L 1$ has been reported to induce hypertrophic differentiation, apoptosis and calcification in chondrocytes (31); CCL2 has been implicated in the lung recruitment of leukocytes, natural killer cells and T cells (32), and has been demonstrated to enhance nociception (33). Amin and Islam (34) reported that in human OA chondrocytes, the expression of
CXCL1 is increased by 38 -fold, and the expression of CCL2 is increased by 53 -fold. Stone et al (35) reported that the increased expression of $C X C L 1$ is associated with discoid meniscus injuries during OA development. In addition, 3 KEGG pathways were revealed to be enriched in the 17 overlapping DEGs, including the chemokine signaling pathway, cytokine-cytokine receptor interaction and the NOD-like receptor signaling pathway. Chemokines are critical for the perpetuation of the inflammatory response, via attracting proinflammatory cells to the inflamed joints (36). Several chemokines have exhibited proinflammatory and angiogenic properties in $\mathrm{OA}$ and rheumatoid arthritis (RA) studies (37). Zhang et al (38) demonstrated that the 
Table IV. Top 40 most significant miRNA-mRNA regulatory pairs for the overlapping differentially expressed genes, according to the predicted database number.

\begin{tabular}{|c|c|c|c|c|c|}
\hline Gene & miRNA & Database number & Gene & miRNA & Database number \\
\hline BASP1 & hsa-miR-150 & 9 & CLEC4D & hsa-miR-502-3p & 6 \\
\hline BASP1 & hsa-miR-7 & 8 & BASP1 & hsa-miR-7 & 6 \\
\hline BASP1 & hsa-miR-200b & 8 & CCL2 & hsa-miR-33b & 6 \\
\hline BASP1 & hsa-miR-200c & 7 & CLEC4D & hsa-miR-607 & 6 \\
\hline CCL7 & hsa-miR-23a & 7 & BASP1 & hsa-miR-298 & 6 \\
\hline CLEC4D & hsa-miR-20a & 7 & BASP1 & hsa-miR-429 & 6 \\
\hline CLEC4D & hsa-miR-106a & 7 & CLEC4D & hsa-miR-18b & 6 \\
\hline CLEC4D & hsa-miR-106b & 7 & CLEC4D & hsa-miR-520e & 6 \\
\hline CCL7 & hsa-miR-23b & 7 & BASP1 & hsa-miR-767-5p & 6 \\
\hline CCL2 & hsa-miR-1 & 7 & CLEC4D & hsa-miR-548d-3p & 6 \\
\hline BASP1 & hsa-miR-635 & 7 & BASP1 & hsa-miR-96 & 6 \\
\hline CCL2 & hsa-miR-527 & 6 & BASP1 & hsa-miR-623 & 6 \\
\hline TINAGL1 & hsa-miR-665 & 6 & CCL2 & hsa-miR-506 & 6 \\
\hline CLEC4D & hsa-miR-502-5p & 6 & CCL2 & hsa-miR-33a & 6 \\
\hline BASP1 & hsa-miR-7 & 6 & CLEC4D & hsa-miR-20b & 6 \\
\hline CLEC4D & hsa-miR-568 & 6 & BASP1 & hsa-miR-552 & 6 \\
\hline BASP1 & hsa-miR-142-5p & 6 & CLEC4D & hsa-miR-520f & 6 \\
\hline ARG1 & hsa-miR-648 & 6 & ARG1 & hsa-miR-23b & 6 \\
\hline BASP1 & hsa-miR-765 & 6 & CCL2 & hsa-miR-495 & 6 \\
\hline CLEC4D & hsa-miR-450b-5p & 6 & TINAGL1 & hsa-miR-9 & 6 \\
\hline
\end{tabular}

mi, micro.

Table V. Top 40 most significant nodes, according to the intimate connections with other nodes in the miRNA-mRNA regulatory network.

\begin{tabular}{lclc}
\hline Node & Degree & \multicolumn{1}{c}{ Node } & Degree \\
\hline CLEC4D & 78 & hsa-miR-548d-3p & 2 \\
BASP1 & 38 & hsa-miR-495 & 2 \\
CCL7 & 35 & hsa-miR-494 & 2 \\
TINAGL1 & 29 & hsa-miR-613 & 2 \\
CCL2 & 22 & hsa-miR-206 & 2 \\
ARG1 & 19 & hsa-miR-637 & 2 \\
CXCL1 & 10 & hsa-miR-374a & 2 \\
IL1RN & 7 & hsa-miR-374b & 2 \\
EPHX1 & 4 & hsa-miR-944 & 2 \\
hsa-miR-7 & 4 & hsa-miR-576-5p & 2 \\
hsa-miR-380 & 3 & hsa-miR-485-3p & 2 \\
hsa-miR-586 & 3 & hsa-miR-186 & 2 \\
hsa-miR-587 & 3 & hsa-miR-590-3p & 2 \\
hsa-miR-650 & 3 & hsa-miR-570 & 2 \\
hsa-miR-23a & 2 & hsa-miR-432 & 2 \\
hsa-miR-23b & 2 & hsa-miR-539 & 2 \\
hsa-miR-1 & 2 & hsa-miR-493 & 2 \\
hsa-miR-765 & 2 & hsa-miR-938 & 2 \\
hsa-miR-607 & 2 & hsa-miR-300 & 2 \\
hsa-miR-767-5p & 2 & hsa-miR-548c-3p & 2 \\
\hline
\end{tabular}

miR, microRNA. chemokine signaling pathway was involved in CCL2 expression in tissues isolated from patients with RA, which may contribute to the chronic inflammation associated with RA. Cytokine-cytokine receptor interactions have been reported to serve critical roles in OA pathophysiology (39). The NOD-like receptor has been demonstrated to participate in inflammatory responses in various diseases and conditions, including cholesteatoma, wound healing and autoimmune encephalomyelitis (40-42). The results of the present study suggested that the enriched GO terms and KEGG pathways may be closely associated with the pathogenesis and progression of OA, and thus they may have potential as novel therapeutic targets for the treatment of patients with OA.

Following the construction of the miRNA-mRNA regulatory network, C-type lectin domain family 4, member D (CLEC4D) was identified as a major miRNA target, and had the highest node degree, thus suggesting that it may serve a critical role during OA pathogenesis. CLEC4D codes for a C-type lectin receptor, which recognizes trehalose 6,6'-dimycolate, a mycobacterial cell wall component, and induces potent innate immune responses (43). Since OA is a type of autoimmune disorder, CLEC4D may be implicated in its development and progression. Steichen et al (44) reported that CLEC4D serves a protective role during the resolution of Gram-negative-induced pneumonia. In the present study, hsa-miR-7 was identified as the miRNA regulating the most mRNAs in the constructed network, thus suggesting that hsa-miR-7 may be involved in the pathogenesis and progression of $\mathrm{OA}$; however further 
studies are required to investigate the roles of hsa-miR-7, and other regulatory miRNAs, in the mechanisms underlying the development of OA.

In conclusion, the present study identified potential genes, including CLEC4D, CXCL1 and CCL2, and pathways, including chemokine signaling pathway, cytokine-cytokine receptor interaction and NOD-like receptor signaling pathway, which may be implicated in the pathogenesis and progression of OA. These findings may help elucidate the molecular mechanisms underlying OA pathophysiology, and may be useful for the development of novel therapeutic targets for the treatment of patients with OA.

\section{Acknowledgements}

The authors thank the Special Support Program of Talent Development, Tianjin, China.

\section{References}

1. Poulet B and Staines KA: New developments in osteoarthritis and cartilage biology. Curr Opin Pharmacol 28: 8-13, 2016.

2. Glyn-Jones S, Palmer AJ, Agricola R, Price AJ, Vincent TL, Weinans H and Carr AJ: Osteoarthritis. Lancet 386: 376-387, 2015.

3. Lee KM, Chung CY, Sung KH, Lee SY, Won SH, Kim TG, Choi Y, Kwon SS, Kim YH and Park MS: Risk factors for osteoarthritis and contributing factors to current arthritic pain in South Korean older adults. Yonsei Med J 56: 124-131, 2015.

4. Silverwood V, Blagojevic-Bucknall M, Jinks C, Jordan JL, Protheroe $\mathrm{J}$ and Jordan KP: Current evidence on risk factors for knee osteoarthritis in older adults: A systematic review and meta-analysis. Osteoarthritis Cartilage 23: 507-515, 2015.

5. Kodadek M: Managing osteoarthritis. Nurs Womens Health 19: 71-76, 2015 .

6. Yoshimura N, Muraki S, Oka H, Mabuchi A, En-Yo Y, Yoshida M, Saika A, Yoshida H, Suzuki T, Yamamoto S, et al: Prevalence of knee osteoarthritis, lumbar spondylosis and osteoporosis in Japanese men and women: The research on osteoarthritis/osteoporosis against disability study. J Bone Miner Metab 27: 620-628, 2009.

7. Jorge RT, Souza MC, Chiari A, Jones A, Fernandes Ada R, Lombardi Júnior I and Natour J: Progressive resistance exercise in women with osteoarthritis of the knee: A randomized controlled trial. Clin Rehabil 29: 234-243, 2015.

8. Niessen LC and Fedele DJ: Older adults--implications for private dental practitioners. J Calif Dent Assoc 33: 695-703, 2005.

9. Felson DT: Clinical practice. Osteoarthritis of the knee. N Engl J Med 354: 841-848, 2006.

10. Feeley BT, Gallo RA, Sherman S and Williams RJ: Management of osteoarthritis of the knee in the active patient. J Am Acad Orthop Surg 18: 406-416, 2010.

11. Yu SP and Hunter DJ: Managing osteoarthritis. Aust Prescr 38 $115-119,2015$.

12. Berenbaum F: Osteoarthritis as an inflammatory disease (osteoarthritis is not osteoarthrosis!). Osteoarthritis Cartilage 21: 16-21, 2013.

13. Cao K, Wei L, Zhang Z, Guo L, Zhang C, Li Y, Sun C, Sun X, Wang S, Li P and Wei X: Decreased histone deacetylase 4 is associated with human osteoarthritis cartilage degeneration by releasing histone deacetylase 4 inhibition of runt-related transcription factor-2 and increasing osteoarthritis-related genes: A novel mechanism of human osteoarthritis cartilage degeneration. Arthritis Res Ther 16: 491, 2014.

14. Yu XM, Meng HY, Yuan XL, Wang Y, Guo QY, Peng J, Wang AY and Lu SB: MicroRNAs' involvement in osteoarthritis and the prospects for treatments. Evid Based Complement Alternat Med 2015: 236179, 2015

15. Evans CH, Gouze JN, Gouze E, Robbins PD and Ghivizzani SC: Osteoarthritis gene therapy. Gene Ther 11: 379-389, 2004.

16. Jacobs JP, Ortiz-Lopez A, Campbell JJ, Gerard CJ, Mathis D and Benoist C: Deficiency of CXCR2, but not other chemokine receptors, attenuates autoantibody-mediated arthritis in a murine model. Arthritis Rheum 62: 1921-1932, 2010.
17. Gautier L, Cope L, Bolstad BM and Irizarry RA: Affy-analysis of Affymetrix GeneChip data at the probe level. Bioinformatics 20: 307-315, 2004.

18. Smyth G and Smyth GK: Limma: Linear models for microarray data. Bioinformatics and Computational Biology Solution Using $\mathrm{R}$ and Bioconductor, 2013.

19. Dennis G Jr, Sherman BT, Hosack DA, Yang J, Gao W, Lane HC and Lempicki RA: DAVID: Database for annotation, visualization and integrated discovery. Genome Biol 4: P3, 2003.

20. Dweep H, Sticht C, Pandey P and Gretz N: miRWalk--database: Prediction of possible miRNA binding sites by "walking" the genes of three genomes. J Biomed Inform 44: 839-847, 2011.

21. Shannon P, Markiel A, Ozier O, Baliga NS, Wang JT, Ramage D, Amin N, Schwikowski B and Ideker T: Cytoscape: A software environment for integrated models of biomolecular interaction networks. Genome Res 13: 2498-2504, 2003.

22. Panoutsopoulou K, Southam L, Elliott KS, Wrayner N, Zhai G, Beazley C, Thorleifsson G, Arden NK, Carr A, Chapman K, et al: Insights into the genetic architecture of osteoarthritis from stage 1 of the arcOGEN study. Ann Rheum Dis 70: 864-867, 2011.

23. Loughlin J: Genetic contribution to osteoarthritis development: Current state of evidence. Curr Opin Rheumatol 27: 284-288, 2015.

24. de Lange-Brokaar BJ, Ioan-Facsinay A, van Osch GJ, Zuurmond AM, Schoones J, Toes RE, Huizinga TW and Kloppenburg M: Synovial inflammation, immune cells and their cytokines in osteoarthritis: A review. Osteoarthritis Cartilage 20: 1484-1499, 2012

25. Sohn DH, Sokolove J, Sharpe O, Erhart JC, Chandra PE, Lahey LJ, Lindstrom TM, Hwang I, Boyer KA, Andriacchi TP and Robinson WH: Plasma proteins present in osteoarthritic synovial fluid can stimulate cytokine production via Toll-like receptor 4. Arthritis Res Ther 14: R7, 2012.

26. Sakkas LI, Scanzello C, Johanson N, Burkholder J, Mitra A, Salgame P, Katsetos CD and Platsoucas CD: T cells and T-cell cytokine transcripts in the synovial membrane in patients with osteoarthritis. Clin Diagn Lab Immunol 5: 430-437, 1998.

27. Wang Q, Rozelle AL, Lepus CM, Scanzello CR, Song JJ, Larsen DM, Crish JF, Bebek G, Ritter SY, Lindstrom TM, et al: Identification of a central role for complement in osteoarthritis. Nat Med 17: 1674-1679, 2011.

28. Kandahari AM, Yang X, Dighe AS, Pan D and Cui Q: Recognition of immune response for the early diagnosis and treatment of osteoarthritis. J Immunol Res 2015: 192415, 2015.

29. Haseeb A and Haqqi TM: Immunopathogenesis of osteoarthritis. Clin Immunol 146: 185-196, 2013.

30. Calich AL, Domiciano DS and Fuller R: Osteoarthritis: Can anti-cytokine therapy play a role in treatment?. Clin Rheumatol 29: 451-455, 2010.

31. Merz D, Liu R, Johnson K and Terkeltaub R: IL-8/CXCL8 and growth-related oncogene alpha/CXCL1 induce chondrocyte hypertrophic differentiation. J Immuno 171: 4406-4415, 2003.

32. Lehmann MH, Torres-Domínguez LE, Price PJ, Brandmüller C, Kirschning CJ and Sutter G: CCL2 expression is mediated by type I IFN receptor and recruits NK and T cells to the lung during MVA infection. J Leukoc Biol 99: 1057-1064, 2016.

33. Van Steenwinckel J, Auvynet C, Sapienza A, Reaux-Le Goazigo A, Combadière C and Melik Parsadaniantz S: Stromal cell-derived CCL2 drives neuropathic pain states through myeloid cell infiltration in injured nerve. Brain Behav Immun 45: 198-210, 2015.

34. Amin AR and Islam AB: Genomic analysis and differential expression of HMG and S100A family in human arthritis: Upregulated expression of chemokines, IL-8 and nitric oxide by HMGB1. DNA Cell Biol 33: 550-565, 2014.

35. Stone AV, Loeser RF, Vanderman KS, Long DL, Clark SC and Ferguson CM: Pro-inflammatory stimulation of meniscus cells increases production of matrix metalloproteinases and additional catabolic factors involved in osteoarthritis pathogenesis. Osteoarthritis Cartilage 22: 264-274, 2014.

36. Hampel U, Sesselmann S, Iserovich P, Sel S, Paulsen F and Sack R: Chemokine and cytokine levels in osteoarthritis and rheumatoid arthritis synovial fluid. J Immunol Methods 396: 134-139, 2013

37. Szekanecz Z, Kim J and Koch AE: Chemokines and chemokine receptors in rheumatoid arthritis. Semin Immunol 15: 15-21, 2003. 
38. Zhang L, Yu M, Deng J, Lv X, Liu J, Xiao Y, Yang W, Zhang Y and Li C: Chemokine signaling pathway involved in CCL2 expression in patients with rheumatoid arthritis. Yonsei Med J 56: 1134-1142, 2015.

39. Cui S, Zhang X, Hai S, Lu H, Chen Y, Li C, Tong P, Lu F and Yuan Z: Molecular mechanisms of osteoarthritis using gene microarrays. Acta Histochem 117: 62-68, 2015.

40. Leichtle A, Klenke C, Ebmeyer J, Daerr M, Bruchhage KL, Hoffmann AS, Ryan AF, Wollenberg B and Sudhoff H: NOD-like receptor signaling in cholesteatoma. Biomed Res Int 2015: 408169, 2015.

41. Weinheimer-Haus EM, Mirza RE and Koh TJ: Nod-like receptor protein-3 inflammasome plays an important role during early stages of wound healing. PLoS One 10: e0119106, 2015.
42. Gharagozloo M, Mahvelati TM, Imbeault E, Gris P, Zerif E, Bobbala D, Ilangumaran S, Amrani A and Gris D: The nod-like receptor, Nlrp12, plays an anti-inflammatory role in experimental autoimmune encephalomyelitis. J Neuroinflammation 12: 198, 2015.

43. Zhao XQ, Zhu LL, Chang Q, Jiang C, You Y, Luo T, Jia XM and Lin X: C-type lectin receptor dectin-3 mediates trehalose 6,6'-dimycolate (TDM)-induced Mincle expression through CARD9/Bc110/MALT1-dependent nuclear factor (NF)- $\kappa \mathrm{B}$ activation. J Biol Chem 289: 30052-30062, 2014.

44. Steichen AL, Binstock BJ, Mishra BB and Sharma J: C-type lectin receptor Clec4d plays a protective role in resolution of Gram-negative pneumonia. J Leukoc Biol 94: 393-398, 2013. 